\title{
Characterization and Etiopathogenic Approach of Pediatric Renal Biopsy Patients in a Colombian Medical Center from 2007-2017
}

\author{
Mayerly Prada Rico $D^{1},{ }^{1}$ Carmen Inés Rodríguez Cuellar, ${ }^{2}$ \\ Monica Fernandez Hernandez $(\mathbb{D}),{ }^{3}$ Luz Stella González Chaparro $\mathbb{D D}^{1},{ }^{1}$ \\ Olga Lucía Prado Agredo $\mathbb{D},{ }^{1}$ and Ricardo Gastelbondo Amaya $\mathbb{D}^{1}$ \\ ${ }^{1}$ Pediatric Nephrology Division, Pediatrics Department, Fundación Cardioinfantil, Bogotá, Cundinamarca, Colombia \\ ${ }^{2}$ Pediatric Emergency Care Division, Pediatrics Department, Fundación Cardioinfantil, Bogotá, Cundinamarca, Colombia \\ ${ }^{3}$ Pediatric Endocrinology Division, Pediatrics Department, Fundación Cardioinfantil, Bogotá, Cundinamarca, Colombia
}

Correspondence should be addressed to Mayerly Prada Rico; mpradar@cardioinfantil.org

Received 26 February 2018; Accepted 4 June 2018; Published 28 June 2018

Academic Editor: Suresh C. Tiwari

Copyright (C) 2018 Mayerly Prada Rico et al. This is an open access article distributed under the Creative Commons Attribution License, which permits unrestricted use, distribution, and reproduction in any medium, provided the original work is properly cited.

\begin{abstract}
Introduction. Renal biopsy is the principal instrument to evaluate the diagnosis and prognosis of children with kidney disease. There are relatively few studies establishing epidemiology of its findings in the pediatric population. Methods. A descriptive study was conducted to describe characteristics of pediatric patients who had undergone a renal biopsy over the last 10 years in a national reference center, trying to accomplish an etiopathogenic approach of biopsy findings. Results. 241 patients were included. Most frequent indications were nephrotic syndrome (34.1\%) and systemic disease with renal involvement (30.2\%). The most prevalent biopsy diagnosis was glomerulonephritis (44\%) and among these patients, glomerulonephritis mediated by immune complexes was the most frequent pathogenic type (90.5\%). When the biopsy was indicated for proteinuria plus hematuria and systemic disease with renal involvement, the most frequent biopsy diagnosis was glomerulonephritis (60 and $85 \%$, respectively). For isolated hematuria, the predominant biopsy diagnosis was inherited diseases of the glomerular basement membrane (70\%) and for nephrotic syndrome, podocytopathy (82\%). Glomerulonephritis was more frequent in patients older than $10 \mathrm{yrs}(65 \%)$ and the rate of postbiopsy major complications was low (1.2\%). Conclusion. Immune complex glomerulonephritis was the most frequent histological finding, differing from previous reports. To our knowledge this is the first description that classifies biopsy findings according to the probable pathogenic mechanism.
\end{abstract}

\section{Introduction}

Percutaneous renal biopsy allows direct study of renal pathology [1]. Although many pediatric patients with kidney diseases can be properly diagnosed without it, in some situations biopsies are not only a diagnostic resource. Findings also determine prognosis and guide treatments $[2,3]$. In order to establish an adequate diagnostic and therapeutic approach to kidney disease based on biopsy histological findings, it is essential to recognize the pathogenic mechanisms involved, improving the understanding of kidney disease [2]. Mayo Clinic 2015 Nephrologists and Renal Pathologists Consensus is an example of a diagnostic and classification system of glomerulonephritis based on etiology and pathogenesis [4].

Since the first renal biopsy description in 1934 [5], different series have been published describing the indications, histopathological findings, and complications in children [2, 6-23]. However, an etiopathogenic classification of biopsy findings has not been performed to date. Herein, we attempt to accomplish such an approach.

\section{Materials and Methods}

Retrospective review of clinical and pathology reports of under 18 yrs patients who underwent renal biopsy between 
TABLE 1: Histopathological findings classification.

\begin{tabular}{lccc}
\hline Category & Pathogenic type & Specific disease entity & Scores or class \\
\hline Glomerulonephritis (GN) & Immune-complex GN & $\begin{array}{c}\text { LN, IgA Nephropathy, MPGN } \\
\text { Immunoglobulin positive, Post } \\
\text { infectious acute GN } \\
\text { aHUS, C3GN, DDD }\end{array}$ & $\begin{array}{c}\text { Oxford/MEST score for IgA } \\
\text { Nephropathy, ISN/RPS score for } \\
\text { NL }\end{array}$ \\
$\begin{array}{l}\text { Complement Mediated GN } \\
\text { Pauci-immune GN }\end{array}$ & MPO-ANCA GN & $\begin{array}{c}\text { Not-otherwise-specified, cellular, } \\
\text { collapsing disease, tip and } \\
\text { perihilar variants for FSGS }\end{array}$ \\
$\begin{array}{l}\text { Basement membrane } \\
\text { disease (BMD) }\end{array}$ & Primary NS & MCD, FSGS, MN & TMB, AS \\
Tubulointerstitial disease & Glomerular Basement & & Membrane \\
Acute TIN & Chronic TIN & & \\
Others & Nonspecific findings & & \\
& Normal & & \\
\hline
\end{tabular}

LN: Lupus Nephritis; MPGN: Membranoproliferative Glomerulonephritis; aHUS: Atypical Hemolytic Uremic Syndrome; C3GN: C3 Glomerulonephritis; DDD: Dense Deposit Disease; MPO: Myeloperoxidase; ANCA: Anti-neutrophil Cytoplasmic Antibodies; NS: Nephrotic Syndrome; MCD: Minimal Change Disease; FSGS: Focal Segmental Glomerulosclerosis; MN: Membranous Nephropathy; TMB: Thin Membrane Disease; AS: Alport's Syndrome; TIN: Tubulointerstitial Nephritis; MEST: mesangial hypercellularity (M), segmental sclerosis (S), interstitial fibrosis/tubular atrophy (T) lesions; ISN/RPS: International Society of Nephrology/ Renal Pathology Society

January 2007 and May 2017 at Fundación Cardioinfantil, Bogota, Colombia. Demographics, clinical variables, indication of renal biopsy, histological findings, and biopsy complications were analyzed.

Renal biopsy indications were nephrotic syndrome, systemic disease with renal involvement, isolated subnephrotic proteinuria, proteinuria and hematuria, isolated hematuria, glomerular filtration rate impairment without a known cause, isolated nephrotic proteinuria, and nephritic syndrome. Isolated subnephrotic proteinuria was defined as the presence of 4 to $40 \mathrm{mg} / \mathrm{m}^{2} /$ hour in a 24 -hour urine collection or a urinary protein/creatinine ratio between 0.2 and 2 in an isolated sample without other symptoms (edema, dyslipidemia, hypoalbuminemia, hematuria, etc.). Isolated hematuria was defined as the presence of macro or microscopic hematuria without other symptoms (proteinuria, edema, hypertension, decreased glomerular filtration rate, etc.).

Histological findings were classified as detailed in Table 1. Biopsy complications were classified as follows: (1) major: if they involved additional interventions or outcomes (resuscitation, transfusion of blood components, surgery, prolonged hospital stay, readmission, death, infection, or macroscopic hematuria); (2) minors: if they did not involve additional interventions or outcomes.

Patients were admitted on the previous biopsy day and underwent a clinical evaluation including: clinical history, physical examination, and complementary bloodwork (complete blood count, partial thromboplastin time, and prothrombin time). Postbiopsy evaluation was performed as follows: (1) Complete blood count 6 hours after the intervention or earlier if required (hemodynamic instability, macroscopic hematuria, etc.). (2) Hourly vital signs and macroscopic hematuria surveillance over the first 6 to 12 hours. (3) Kidney ultrasound taken after 24 hours.
All the biopsies were ultrasound guided and performed by interventional radiologists under general anesthesia. An automatic percutaneous biopsy instrument and $18 \mathrm{G}$ needles were used for all patients regardless of their stature.

Samples were studied under light microscopy, immunofluorescence, and electron microscopy.

2.1. Statistical Analysis. Description of demographics, clinical findings, and relevant variables related to the histopathological findings, through relative frequency measurements. Comparative tables and graphs with frequency distribution.

All analyses were performed using STATA version 9 software (StataCorp LP, College Station, TX).

\section{Results}

Total number of patients that were included in biopsy was 241; 58\% (140/241) females, 78.8\% (190/241) original from Colombia's central region. Renal biopsy mean age was 11 yrs (4.3 SDS).

$27.8 \%(67 / 241)$ had impaired glomerular filtration rate and $80.4 \%(194 / 241)$ comorbidities such as acute kidney injury (13.7\%; 33/241), systemic diseases (29.8\%; 72/241), coagulopathy $(2.9 \%$; 7/241), high blood pressure $(32.7 \%$; $79 / 241)$, and solitary kidney $(1.6 \% ; 4 / 241)$. Table 2 shows kidney disease stage and the associated systemic disease.

3.1. Renal Biopsy Indications. Renal biopsy indications are illustrated in Figure 1. Among nephrotic syndrome patients $46.3 \%(38 / 82)$ had steroid-resistant nephrotic syndrome (SRNS), 44\% (36/82) steroid-dependent nephrotic syndrome (SDNS), and 9.7\% (8/82) frequently relapsing nephrotic syndrome (FRNS). Indications frequency according to age is shown in Table 3. Systemic disease with renal involvement 


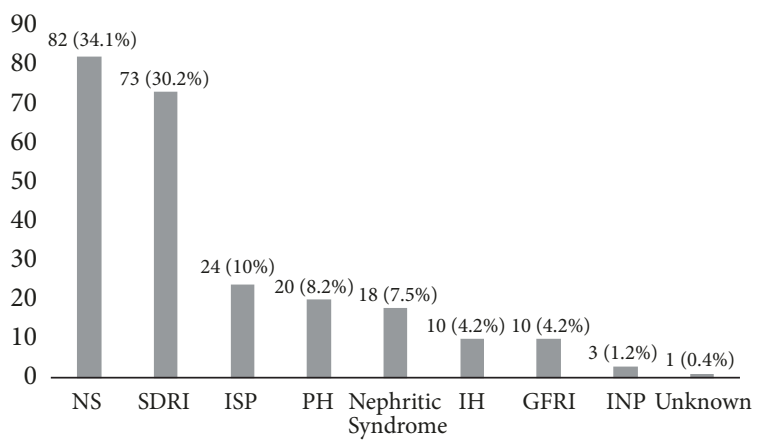

FIGURE 1: Renal biopsy indications. NS: nephrotic syndrome; SDRI: Systemic disease with renal involvement; ISP: isolated subnephrotic proteinuria; $\mathrm{PH}$ : proteinuria and hematuria; $\mathrm{IH}$ : isolated hematuria; GFRI: glomerular filtration rate impairment without a known cause; INP: isolated nephrotic proteinuria.

TABLE 2: Kidney disease stage and associated systemic disease.

\begin{tabular}{lcc}
\hline & & $\mathbf{n}(\%)$ \\
\hline & G1 & $194(80.5)$ \\
Kidney disease stage & G3A & $15(6.2)$ \\
according to KDIGO & G3B & $5(2.1)$ \\
classification & G4 & $3(1.3)$ \\
& G5 & $15(6.2)$ \\
Unknown & $6(2.5)$ \\
& ESL & $3(1.2)$ \\
& SHP & $51(21.2)$ \\
& ITP & $11(4.5)$ \\
& Others & $4(1.7)$ \\
\hline
\end{tabular}

ESL: erythematosus systemic lupus; SHP: Schönlein-Henoch purpura; ITP: immune thrombocytopenic purpura.

TABLE 3: Indications frequency according to age.

\begin{tabular}{lcc}
\hline Indication & $<\mathbf{1 0}$ years & $>$ 10 years \\
& $\mathrm{n}(\%)$ & $\mathrm{n}(\%)$ \\
\hline NS & $48(48 \%)$ & $34(24.1 \%)$ \\
SDRI & $16(16 \%)$ & $57(40.5 \%)$ \\
ISP & $9(9 \%)$ & $15(10.6 \%)$ \\
PH & $3(3 \%)$ & $17(12 \%)$ \\
Nephritic Syndrome & $11(11 \%)$ & $7(5 \%)$ \\
IH & $6(6 \%)$ & $4(2.83 \%)$ \\
GFRI & $4(4 \%)$ & $6(4.3 \%)$ \\
INP & $2(2 \%)$ & $1(0.7 \%)$ \\
Unknown & $1(1 \%)$ & 0 \\
Total & $100(100 \%)$ & $141(100 \%)$ \\
\hline
\end{tabular}

NS: nephrotic syndrome; SDRI: systemic disease with renal involvement; ISP: isolated subnephrotic proteinuria; $\mathrm{PH}$ : proteinuria and hematuria; $\mathrm{IH}$ : isolated hematuria; GFRI: glomerular filtration rate impairment without a known cause; INP: isolated nephrotic proteinuria.

was the most frequent indication in children older than $10 \mathrm{yrs}$ while nephrotic syndrome was the most frequent indication
TABLE 4: Distribution of histopathological findings.

\begin{tabular}{lc}
\hline Histopathological findings & $\mathbf{n}(\mathbf{\%})$ \\
\hline 1. Glomerulonephritis (GN) & $\mathbf{1 0 6}(\mathbf{4 4 )}$ \\
\hline 1.1 Immune-complex GN & $96(39.8)$ \\
LN & $48(19.9)$ \\
IgA Nephropathy & $35(14.5)$ \\
MPGN Immunoglobulin positive & $8(3.3)$ \\
Post infectious acute GN & $5(2)$ \\
1.2 Complement Mediated GN & $9(3.7)$ \\
aHUS & $4(1.6)$ \\
C3GN & $3(1.2)$ \\
DDD & $2(0.8)$ \\
1.3 Pauci-immune GN & $1(0.41)$ \\
MPO-ANCA GN & $1(0.41)$ \\
\hline 2. Podocytopathy & $\mathbf{8 1}(\mathbf{3 3 . 6})$ \\
\hline 2.1 Primary NS & $81(33.6)$ \\
MCD & $47(19.5)$ \\
FSGS & $28(11.6)$ \\
MN & $6(2.48)$ \\
\hline 3. Basement membrane disease & $\mathbf{1 3 ( 5 . 3 )}$ \\
\hline 3.1 Inherited diseases of the glomerular & \\
basement membrane & \\
TMB & $13(5.3)$ \\
\hline 4. Tubulointerstitial disease & $\mathbf{1 4 ( 5 . 8 )}$ \\
\hline Acute TIN & $14(5.8)$ \\
\hline Nothers & $\mathbf{2 7}(\mathbf{1 1 . 2})$ \\
Normal & $18(7.4)$ \\
End Stage kidney & $4(1.6)$ \\
\hline
\end{tabular}

LN: lupus nephritis; MPGN: membranoproliferative glomerulonephritis; aHUS: atypical hemolytic uremic syndrome; C3GN: C3 glomerulonephritis; DDD: dense deposit disease; MPO: myeloperoxidase; ANCA: anti-neutrophil cytoplasmic antibodies; NS: nephrotic syndrome; MCD: minimal change disease; FSGS: focal segmental glomerulosclerosis; MN: membranous nephropathy; TMB: thin membrane disease; TIN: tubulointerstitial nephritis.

in children younger than 10 yrs. Other indications showed a variable distribution between the groups.

3.2. Histopathological Findings. The most frequent biopsy diagnosis was glomerulonephritis (44\%), followed by podocytopathy (33.6\%), basement membrane disease (5.3\%), and tubulointerstitial disease (5.8\%).

Among glomerulonephritis (106/241) the most common pathogenic type was glomerulonephritis mediated by immune complexes $(90.5 \%$; 96/106) and of this, the most common specific entity was lupus nephritis (50\%; 48/96).

In every podocytopathy cases the pathogenic type was primary nephrotic syndrome and of this, the most common specific entity was minimal changes disease (MCD) (58\%; 47/81) (Table 4).

5 out of 48 (10.4\%) lupus nephritis patients were classified as class I or II, $37 / 48(77 \%)$ as class III or IV, $4 / 48(8.3 \%)$ as class V, and $1 / 48(2 \%)$ as class VI. 
Regarding the IgA Nephropathy subgroup of patients, $31.4 \%(11 / 35)$ had a previous medical history of systemic diseases. $72.7 \%$ (8/11) had Schönlein Henoch purpura, 18.1\% $(2 / 11)$ immune thrombocytopenic purpura, and $9.0 \%(1 / 11)$ erythematous systemic lupus. The most frequent Oxford classification was MOSOE0T0 in 28.5\% (10/35) followed by M1S0E1T0 in 14.2\% (5/35) and M1S1E1T0 in 14.2\% (5/35).

Among 28 patients with focal segmental glomerulosclerosis (FSGS) the histopathological variants found were as follows: not-otherwise-specified in $42.8 \%$ (12/28), cellular $39.2 \%$ (11/28), collapsing disease $7.1 \%(2 / 28)$, tip $7.1 \%(2 / 28)$, and perihilar $3.5 \%(1 / 28)$.

\subsection{Renal Biopsy Indications and Histopathological Findings} Correlation. In the subgroup of patients undergoing renal biopsy due to proteinuria and hematuria, systemic disease with renal involvement, and nephritic syndrome, the most frequent biopsy diagnosis was glomerulonephritis that was present in more than $50 \%$ of the cases. Additionally, when the indication was isolated hematuria and nephrotic syndrome, the most common biopsy diagnosis was basement membrane disease and podocytopathy, respectively. On the contrary, for glomerular filtration rate impairment without a known cause and isolated subnephrotic proteinuria patients, the distribution of biopsy diagnoses was variable (Figure 2).

Glomerulonephritis was more frequent in patients with SRNS versus SDNS/FRNS (24\% and 4\%, respectively) and in patients with proteinuria plus hematuria versus isolated hematuria (60\% and $20 \%$, respectively).

When renal biopsy indication was nephrotic syndrome $(n=82)$, the most prevalent specific entity was MCD $(52.4 \%$; $43 / 82)$, followed by FSGS $(25 \% ; 21 / 82)$. However, when analyzing according to corticosteroids response, the FSGS was more frequent in SRNS patients $(39.5 \% ; 15 / 38)$ while MCD in SDNS/FRNS (77.2\%; 34/44) (Figure 3).

In $7 / 10$ isolated hematuria cases, the specific entity was thin basement membrane disease, in 2/10 IgA Nephropathy, and in one case the findings were nonspecific.

As far as biopsy diagnosis according to age group, glomerulonephritis was more frequent in children older than 10 yrs than in children under 10 yrs (Figure 4 ).

3.4. Complications. The only postbiopsy complication was subcapsular hematoma, present in 56 out of 241 cases $(23.2 \%)$. In $54 / 56$ cases $(98.8 \%)$ hematomas were $<2 \mathrm{~cm}$ and there was no need of additional interventions. Due to their size, hemodynamic instability, anemia, and transfusion need, 2/56 cases $(1.2 \%)$ were considered as a major complication. There were no cases of perforation, infection, fistula, or death.

\section{Discussion}

Over the years there is growing interest in recognizing the significance of histomorphological biopsy findings and pathogenic mechanisms involved in diseases affecting glomerular, vascular, or tubulointerstitial kidney structures. Thus, terms such as podocytopathy arise to group those entities in which podocyte biology is affected, as FSGS or MCD [24]. Likewise, membranoproliferative glomerulonephritis classification has been reconsidered, taking into account not only histomorphological findings, but also the presence of immune complex or complement as a pathogenic mechanism $[25,26]$. These new approaches help to understand histopathological lesions, identify new or under diagnosed entities, and establish therapeutic guidelines and further investigations needed [26].

Based on this need, the Mayo Clinic/Renal Pathology Society Consensus Report on Pathologic Classification, Diagnosis, and Reporting of Glomerulonephritis emerged in 2015. This approach includes a primary diagnosis constituted by the pathogenic type, specific entity, injury pattern, and the lesion classification according to the case, followed by a secondary diagnosis, reporting coexisting injuries not necessarily associated with the primary diagnosis [4].

To our knowledge, there are relatively few studies describing renal biopsy indications, findings, and complications epidemiology in pediatrics (Table 5). None of them have a pathogenic approach of biopsy histological findings being this, the first pediatric description trying to achieve it.

Similar to previous pediatric reports, renal biopsy mean age in our study was 9 to 11 years $[6,8-10,13,18,20,22,23]$ and nephrotic syndrome was the most frequent indication $[2,6-$ $15,18,20,21]$. However, unlike other studies $[8,13,22]$ there was a high proportion of patients with systemic disease and the prevalence of isolated hematuria was low.

Past descriptions showed "primary glomerular diseases" as the most frequent biopsy finding, including entities like MCD, FSGS, IgA Nephropathy, among others [2, 6, 817]. Opposite to this, in our study, glomerulonephritis was the most common finding. Considering immune complex, complement, and other immunological mechanisms involved in this entity, the presence of systemic diseases in a significant proportion of the children included seems to be like a plausible explanation (Table 2). However, it is also possible that a pathophysiological approach of biopsy findings made us categorize immune complex glomerulonephritis entities that are traditionally considered as primary.

The finding of lupus nephritis as the most common specific entity among immune complexes mediated glomerulonephritis is striking. Considering that in previous series the most frequent specific entity was IgA Nephropathy $[13,21$, 27]. This may suggest a different epidemiological behavior in our population.

Due to insufficient data in medical records, classification of tubulointerstitial diseases according to pathogenic mechanisms was not possible. This aspect should be taken into account in future descriptions.

This study ratifies variability in glomerulonephritis clinical manifestations $[28,29]$ but shows that glomerulonephritis mediated by immune mechanisms as a finding could be more likely in children with proteinuria and hematuria, systemic disease with renal involvement, nephritic syndrome, and SRNS or being older than 10 yrs, unlike podocytopathies, which were more prevalent in children with SDNS/FRNS and younger than 10 yrs.

In recent years emphasis has been placed on nephrotic syndrome epidemiology changes, due to an increase in FSGS lesions [30, 31]. But, in our series, MCD was the most 


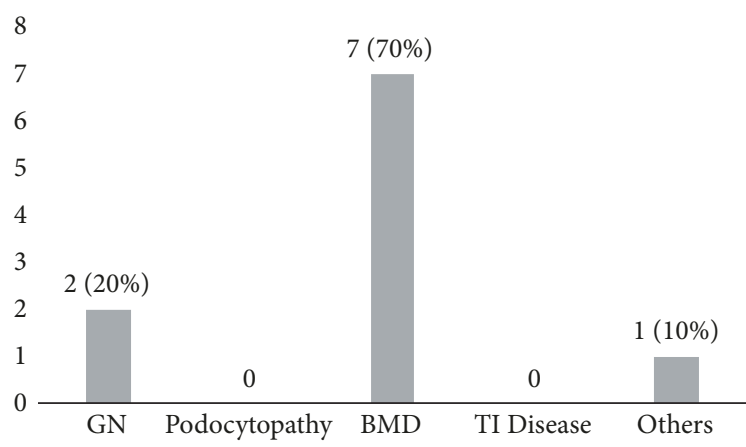

(a)

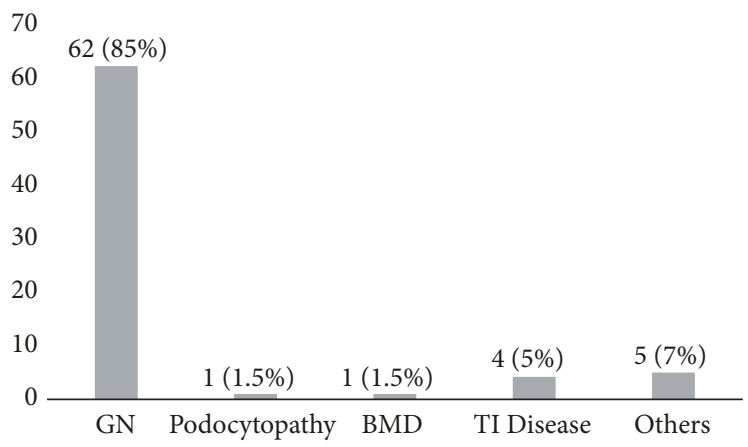

(c)

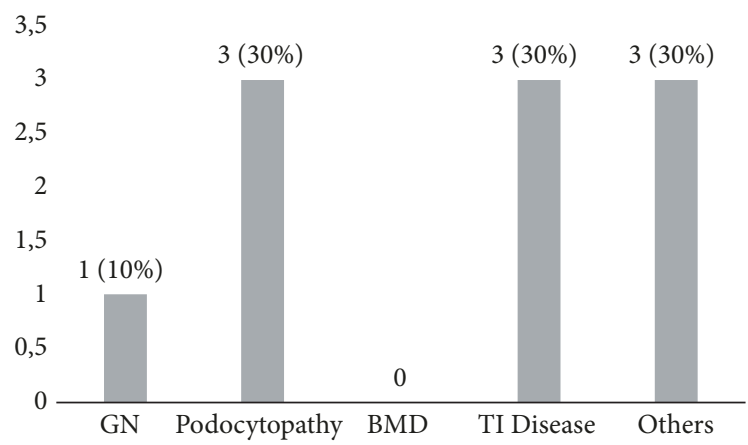

(e)

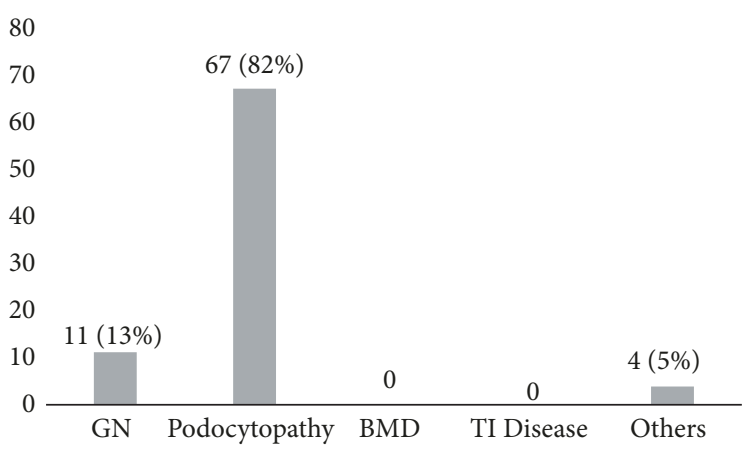

(g)

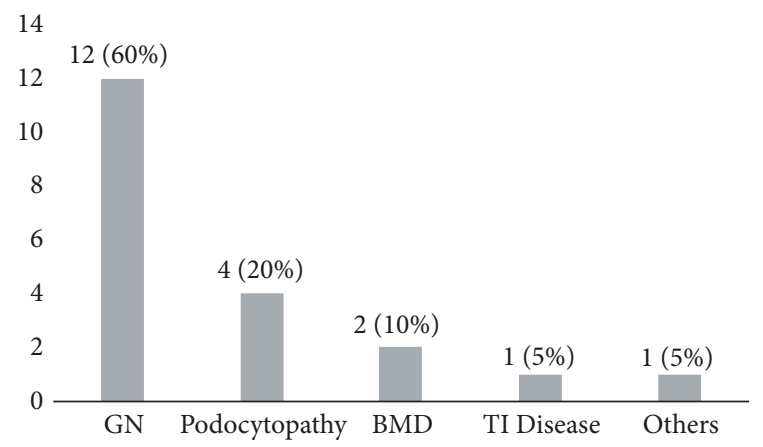

(b)

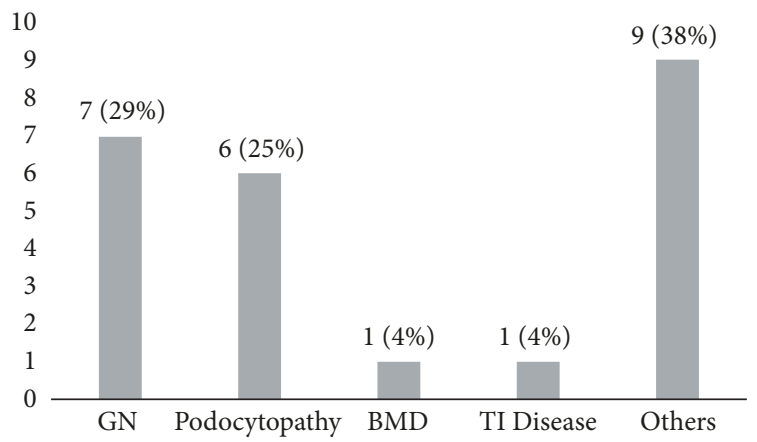

(d)

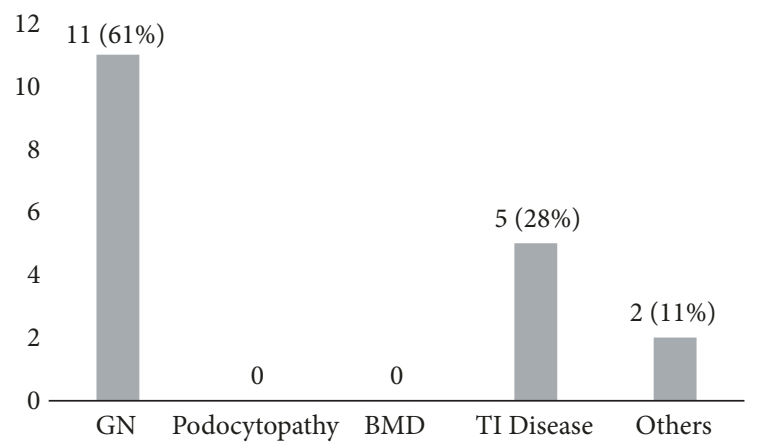

(f)

FIGURE 2: Renal biopsy indications and biopsy diagnoses. (a) Isolated hematuria; (b) proteinuria and hematuria; (c) systemic disease with renal involvement; (d) isolated subnephrotic proteinuria; (e) glomerular filtration rate impairment without a known cause; (f) nephritic syndrome; (g) nephrotic syndrome; GN: glomerulonephritis; TI: tubulointerstitial; BMD: basement membrane disease. 


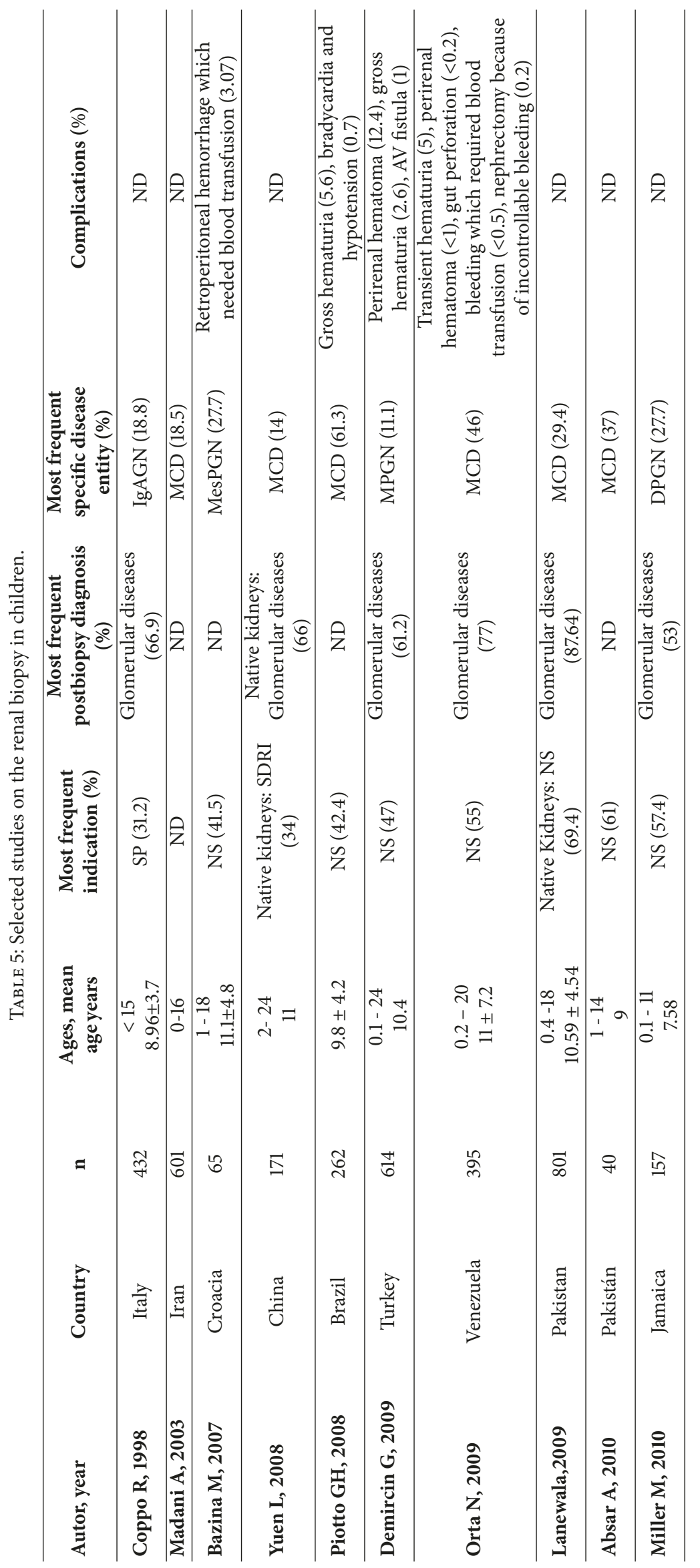




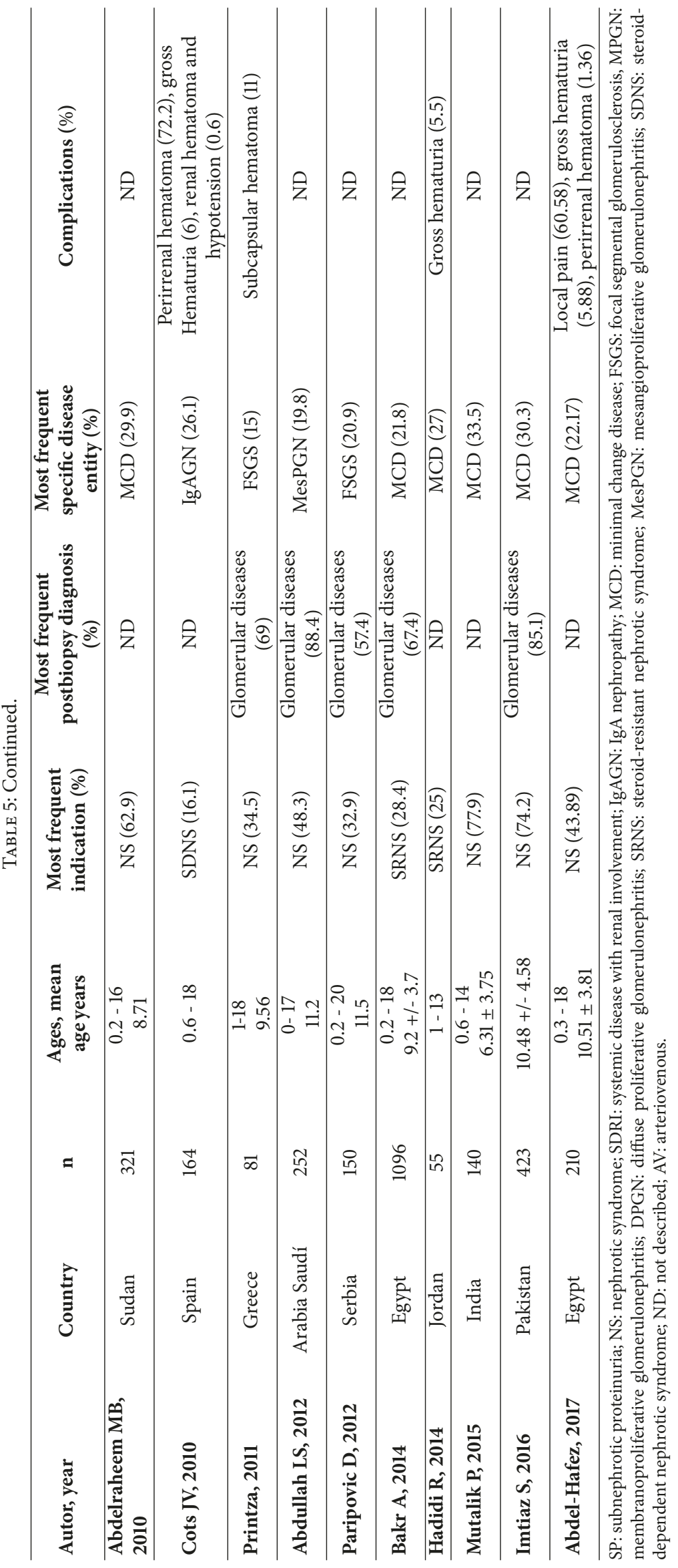




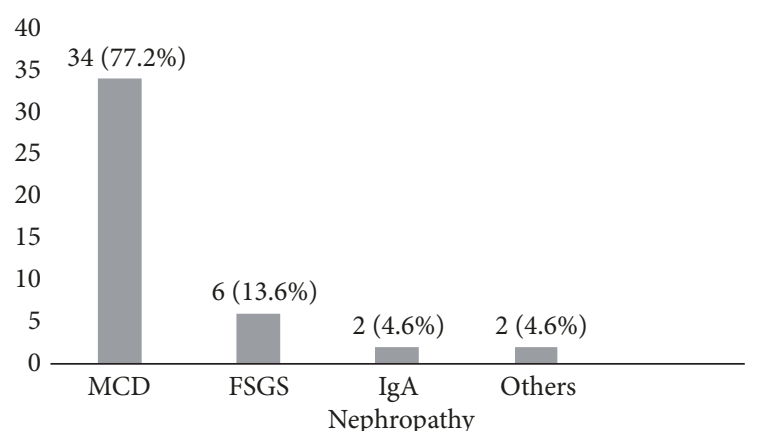

(a)

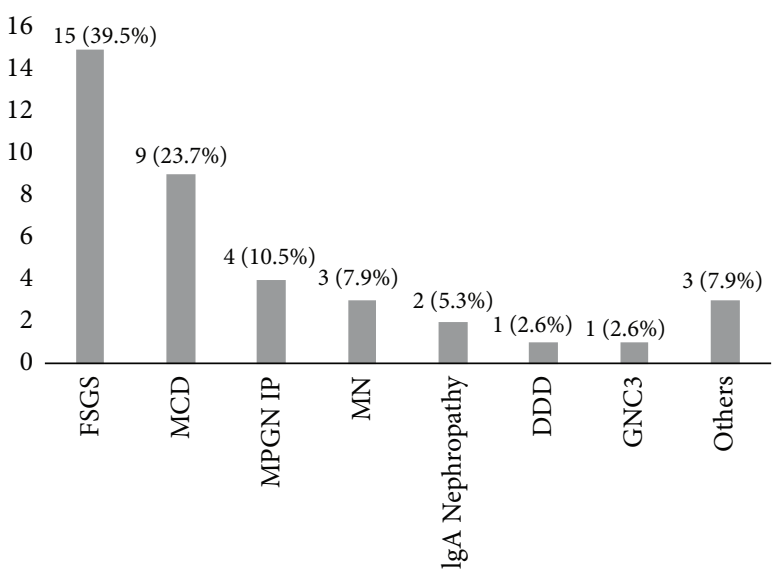

(b)

FIGURE 3: Specific entity according to corticosteroids response. (a) Steroid-dependent or frequently relapsing nephrotic syndrome; (b) steroidresistant nephrotic syndrome; MCD: minimal change disease; FSGS: focal segmental glomerulosclerosis; MPGN IP: membranoproliferative glomerulonephritis immunoglobulin positive; MN: membranous nephropathy; DDD: dense deposit disease; C3GN: C3 glomerulonephritis.

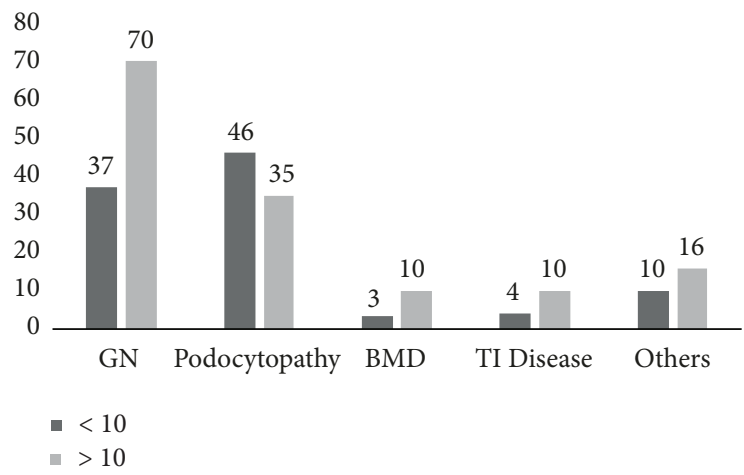

FIGURE 4: Biopsy diagnosis according to age group. GN: glomerulonephritis; TI: tubulointerstitial; BMD: basement membrane disease.

common histopathological lesion. Nonetheless, when analyzing by subgroups according to corticosteroids response, FSGS was more frequent in SRNS while MCD was more frequent in SDNS/FRNS.

The results endorse clinical-pathological correlation relevance, considering agreement in some prebiopsy indications and postbiopsy findings.

Postrenal biopsy complications frequency is variable according to the series previously published (Table 5). In our study, the only complication was subcapsular hematoma in $23.2 \%$ of the cases. Nevertheless, only $1.2 \%$ ( 2 cases) was considered as a major complication. These two cases corresponded to patients admitted to the pediatric intensive care unit due to systemic lupus erythematous debut associated with acute kidney injury and dialysis requirement. Factors as uremia, arterial hypertension, and autoimmune disease may have contributed to this complication development. In the present study, major complications prevalence is much lower compared to other reports 12 to $30.8 \%$ of the cases $[18,32,33]$. This allows us to conclude that, in our center, percutaneous renal biopsy is a safe procedure with a major complications prevalence of less than 5\%, concordant to previously established standards [32].

\section{Conclusion}

In children, classification of histopathological findings in renal biopsy based on the probable etiopathogenic mechanisms constitutes a key instrument not only for an adequate diagnostic and therapeutic approach, but also for the understanding of renal disease epidemiological behavior, according to the population.

\section{Data Availability}

The data used to support the findings of this study are property of Fundación Cardioinfantil. Access to these data will be considered by the author upon request, with permission of Research Department and Institutional Ethics Board Committee.

\section{Ethical Approval}

The study protocol was reviewed and approved by the Fundación Cardioinfantil Ethics Board Committee.

\section{Conflicts of Interest}

The authors declare that they have no conflicts of interest.

\section{Acknowledgments}

The authors thank the patients involved in the study as well as the medical team participating in their health care.

\section{References}

[1] R. Hod Feins, A. Tobar, and M. Davidovits, "Yield and complications of kidney biopsy over two decades in a tertiary pediatric center," Pediatrics International, vol. 59, no. 4, pp. 452-457, 2017. 
[2] G. Demircin, A. Delibaş, K. Bek et al., "A one-center experience with pediatric percutaneous renal biopsy and histopathology in Ankara, Turkey," International Urology and Nephrology, vol. 41, no. 4, pp. 933-939, 2009.

[3] S. Brachemi and G. Bollee, "Renal biopsy practice: What is the gold standard?" World Journal of Nephrology, vol. 3, no. 4, pp. 287-294, 2014.

[4] S. Sethi, M. Haas, G. Markowitz et al., "Mayo Clinic/Renal Pathology Society Consensus Report on Pathologic Classification, Diagnosis, and Reporting of GN," Journal of the American Society of Nephrology, vol. 27, no. 5, pp. 1278-1287, 2016.

[5] R. P. Ball, "Needle (aspiration) Biopsy," Journal of the American Medical Association, vol. 107, no. 17, p. 1381, 1936.

[6] A. Absar, M. Diamond, Y. Sonia et al., "Ten year experience of pediatric kidney biopsies from a single center in Pakistan," Indian Journal of Nephrology, vol. 20, no. 4, pp. 190-192, 2010.

[7] M. Miller, M. Gooden, D. Shah, A. K. Soyibo, J. Williams, and E. N. Barton, "Renal biopsy findings in Jamaican children.," West Indian Medical Journal, vol. 59, no. 3, pp. 325-329, 2010.

[8] N. Orta Sibú, V. Coronel, E. Lara et al., "Biopsia renal en pediatría: análisis de la casuística de 28 años: Hospital de Niños de Valencia-Venezuela," Archivos Venezolanos de Puericultura y Pediatría, vol. 72, no. 4, pp. 123-128, 2009.

[9] A. Bakr, R. Eid, A. Sarhan et al., "Fifteen years of kidney biopsies in children: a single center in Egypt," Saudi Journal of Kidney Disease and Transplantation, vol. 25, no. 6, pp. 1321-1327, 2014.

[10] D. Paripović, M. Kostić, D. Kruščić et al., "Indications and results of renal biopsy in children: A 10-year review from a single center in Serbia," Journal of Nephrology, vol. 25, no. 6, pp. 1054-1059, 2012.

[11] R. Hadidi, M. Hadidi, and M. alDabbas, "Spectrum of biopsyproven kidney disease in children at a Jordanian Hospital," Saudi Journal of Kidney Diseases and Transplantation, vol. 25, no. 3, pp. 680-683, 2014.

[12] S. Imtiaz, K. Nasir, M. Drohlia, B. Salman, and A. Ahmad, "Frequency of kidney diseases and clinical indications of pediatric renal biopsy: A single center experience," Indian Journal of Nephrology, vol. 26, no. 3, pp. 199-205, 2016.

[13] R. Coppo, B. Gianoglio, M. G. Porcellini, and S. Maringhini, "Frequency of renal diseases and clinical indications for renal biopsy in children (report of the italian national registry of renal biopsies in children)," Nephrology Dialysis Transplantation, vol. 13, no. 2, pp. 293-297, 1998.

[14] A. Lanewala, M. Mubarak, F. Akhter, S. Aziz, S. Bhatti, and J. I. Kazi, "Pattern of pediatric renal disease observed in native renal biopsies in Pakistan," Journal of Nephrology, vol. 22, no. 6, pp. 739-746, 2009.

[15] M. B. Abdelraheem, E.-T. M. A. Ali, R. M. Mohamed et al., "Pattern of glomerular diseases in Sudanese children: a clinicopathological study," Saudi Journal of Kidney Diseases and Transplantation, vol. 21, no. 4, pp. 778-783, 2010.

[16] A. Madani, D. Fahimi, S. T. Esfehani et al., "Glomerular diseases in Iranian children: Clinico-pathological correlations," Pediatric Nephrology, vol. 18, no. 9, pp. 925-928, 2003.

[17] P. P. Mutalik, S. K. Pradhan, B. K. Prusty, K. Das, and S. K. Satapathy, "Clinico-pathological correlations of childhood glomerular disease in Eastern India," Sri Lanka Journalof Child Health, vol. 44, no. 1, pp. 31-37, 2015.

[18] M. Bazina, M. Glavina-Durdov, M. Šćukanec-Špoljar et al., "Epidemiology of renal disease in children in the region of Southern Croatia: A 10-year review of regional renal biopsy databases," Medical Science Monitor, vol. 13, no. 4, pp. CR172CR176, 2007.

[19] G. H. M. Piotto, M. C. M. Moraes, D. M. A. C. Malheiros, L. B. Saldanha, and V. H. K. Koch, "Percutaneous ultrasoundguided renal biopsy in children - Safety, efficacy, indications and renal pathology findings: 14-year Brazilian university hospital experience," Clinical Nephrology, vol. 69, no. 6, pp. 417-424, 2008.

[20] L. S. Abdullah, "Histopathological pattern of pediatric renal diseases: a study from a university hospital in western Saudi Arabia," Saudi Journal of Kidney Diseases and Transplantation, vol. 23, no. 2, pp. 377-384, 2012.

[21] J. V. Cots, J. R. Aristizabal, and M. S. Capella, "Resultados de una serie de biopsias renales percutáneas guiadas ecográficamente en una población pediátrica: nuestra experiencia en 15 años," Acta Pediatrica Espanola, vol. 68, no. 10, pp. 493-496, 2010.

[22] L. K. Yuen, W. M. Lai, S. C. Lau, P. C. Tong, K. C. Tse, and M. C. Chiu, "Ten-year review of disease pattern from percutaneous renal biopsy: An experience from a paediatric tertiary renal centre in Hong Kong," Hong Kong Medical Journal, vol. 14, no. 5, pp. 348-355, 2008.

[23] N. Printza, J. Bosdou, A. Pantzaki et al., "Percutaneous ultrasound-guided renal biopsy in children: A single centre experience," Hippokratia, vol. 15, no. 3, pp. 258-261, 2011.

[24] L. Singh, G. Singh, and A. K. Dinda, "Understanding podocytopathy and its relevance to clinical nephrology," Indian Journal of Nephrology, vol. 25, no. 1, pp. 1-7, 2015.

[25] S. Sethi, F. C. Fervenza, Y. Zhang et al., "C3 glomerulonephritis: Clinicopathological findings, complement abnormalities, glomerular proteomic profile, treatment, and follow-up," Kidney International, vol. 82, no. 4, pp. 465-473, 2012.

[26] M. Salvadori and G. Rosso, "Reclassification of membranoproliferative glomerulonephritis: Identification of a new GN: C3GN," World Journal of Nephrology, vol. 5, no. 4, p. 308, 2016.

[27] M. Jiang, Z. Xiao, L. Rong et al., "Twenty-eight-year review of childhood renal diseases from renal biopsy data: A single centre in China," Nephrology, vol. 21, no. 12, pp. 1003-1009, 2016.

[28] A. Perkowska-Ptasinska, A. Bartczak, M. Wagrowska-Danilewicz et al., "Clinicopathologic correlations of renal pathology in the adult population of Poland," Nephrology Dialysis Transplantation, vol. 32, Supplement 2, pp. iii209-iii218, 2017.

[29] F. Rivera, J. M. Lopez-Gomez, and R. Perez-Garcia, "Clinicopathologic correlations of renal pathology in Spain," Kidney International, vol. 66, no. 3, pp. 898-904, 2004.

[30] J. A. Kari, "Changing trends of histopathology in childhood nephrotic syndrome in western Saudi Arabia," Saudi Medical Journal, vol. 23, no. 3, pp. 317-321, 2002.

[31] G. Filler, E. Young, P. Geier, B. Carpenter, A. Drukker, and J. Feber, "Is there really an increase in non-minimal change nephrotic syndrome in children?" American Journal of Kidney Diseases, vol. 42, no. 6, pp. 1107-1113, 2003, Review.

[32] F. Hussain, M. Mallik, S. D. Marks, and A. R. Watson, "Renal biopsies in children: Current practice and audit of outcomes," Nephrology Dialysis Transplantation, vol. 25, no. 2, pp. 485-489, 2010.

[33] R. Sinha, B. Maji, B. Sarkar, and S. Meur, "A prospective audit of complications in 100 consecutive pediatric percutaneous renal biopsies done under real-time ultrasound guidance," Indian Journal of Nephrology, vol. 26, no. 5, pp. 329-334, 2016. 


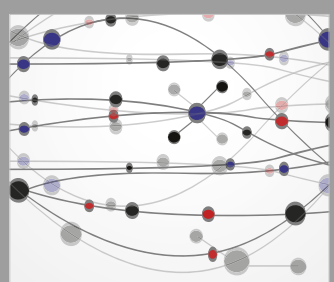

The Scientific World Journal
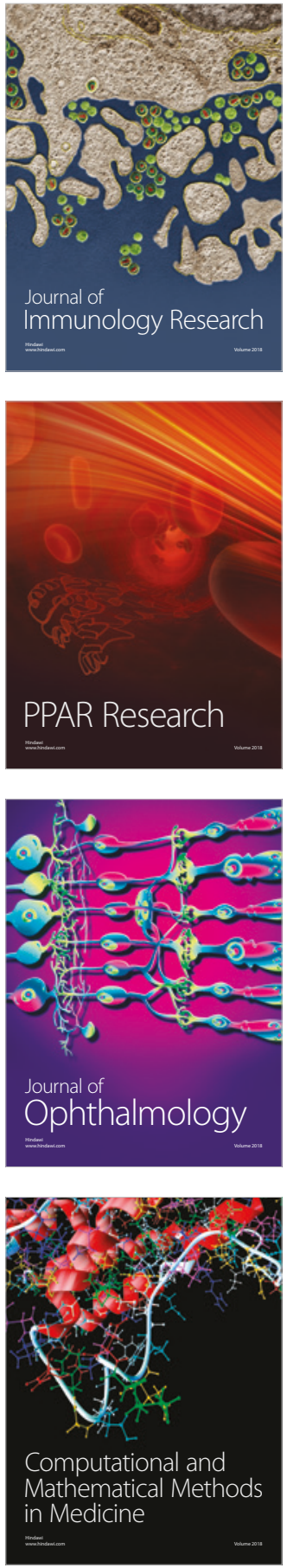

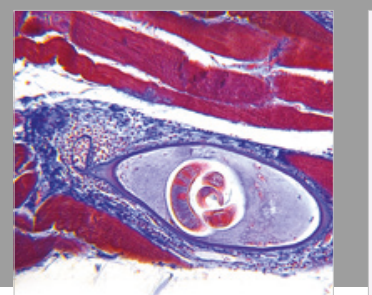

Gastroenterology Research and Practice

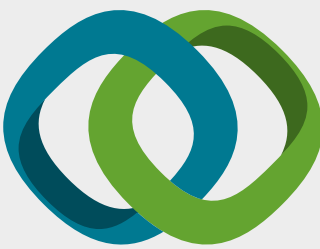

\section{Hindawi}

Submit your manuscripts at

www.hindawi.com
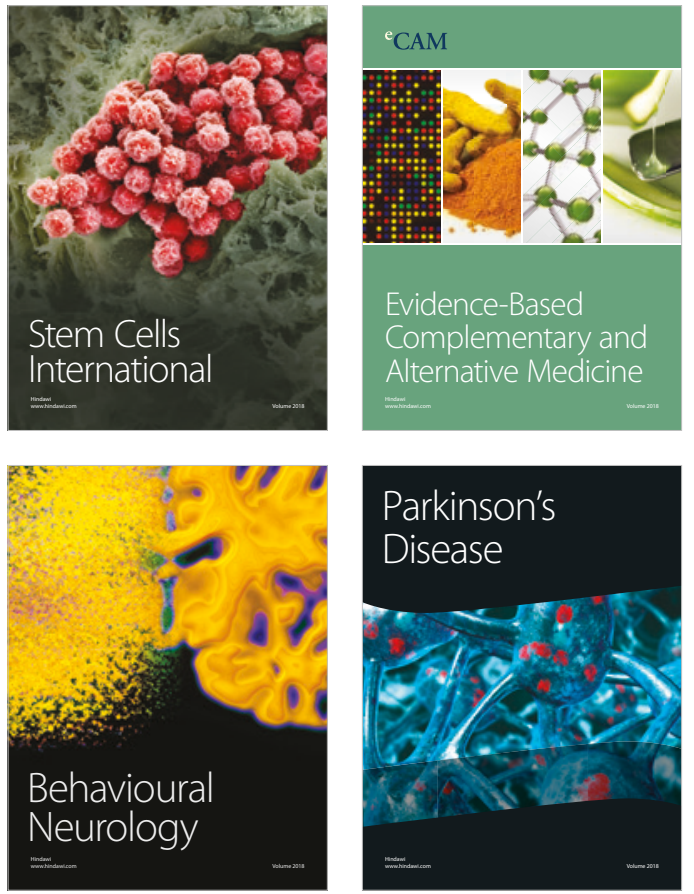

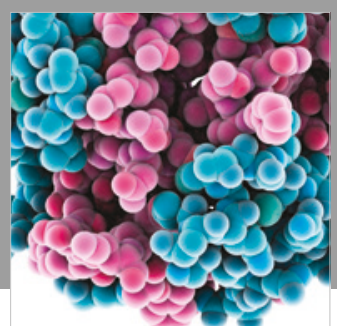

ournal of

Diabetes Research

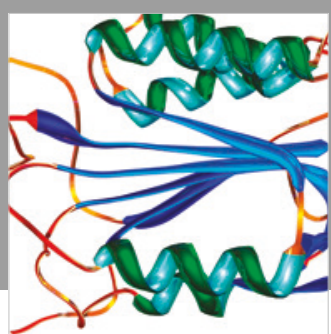

Disease Markers
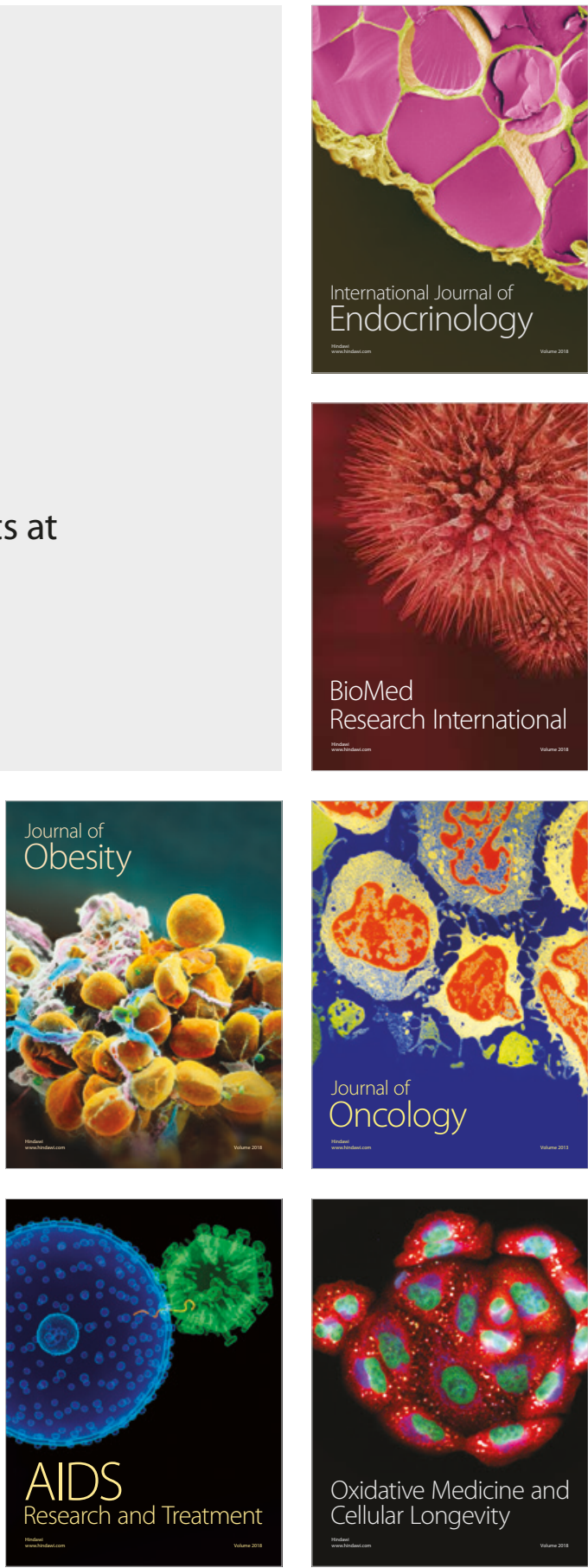